Received: 2017.02.22

Accepted: 2017.03 .28

Published: 2017.12.15

\section{Role of Magnetic Resonance Imaging, Magnetic Resonance Spectroscopy and Transrectal Ultrasound in Evaluation of Prostatic Pathologies with Focus on Prostate Cancer}

\author{
Amol Madanlal Lahoti ${ }^{1 \mathrm{CDEF}}$, Avinash Parshuram Dhok ${ }^{1 \mathrm{AF}}$, \\ Chetana Ramesh Rantnaparkhi ${ }^{1 \mathrm{C}}$, Jitesh Subhash Rawat ${ }^{1 \mathrm{I}}$, \\ Nihar Umakant Chandak ${ }^{2 \mathrm{GF}}$, Hitesh Sharad Tawari ${ }^{3 \mathrm{CD}}$ \\ ${ }^{1}$ Department of Radiodiagnosis, NKP Salve Institute of Medical Sciences and Lata Mangeshkar Hospital, Nagpur, \\ Maharashtra India \\ 2 Department of Surgery, NKP Salve Institute of Medical Sciences and Lata Mangeshkar Hospital, Nagpur, Maharashtra India \\ ${ }^{3}$ Department of Pathology, NKP Salve Institute of Medical Sciences and Lata Mangeshkar Hospital, Nagpur, Maharashtra \\ India
}

Author's address: Amol Madanlal Lahoti, Department of radio-diagnosis, NKP Salve Institute of Medical Sciences and Lata Mangeshkar Hospital Digdoh Hill, Hingna, Nagpur 440017, India, e-mail: amollahoti_203@yahoo.co.in

\section{Summary}

Background: $\quad$ Prostate cancer (PC) is an important medical and socio-economical problem due to its increasing incidence. The development of the prostate specific antigen (PSA) test, and a continuing decrease in the rates of other common neoplasms, such as lung and stomach since mid-1980s, prostate cancer has become one of the most common cancers among men. Prostate cancer (PC) is the second most common cancer in men, preceded only by lung cancer, and its early diagnosis is crucial for a successful treatment, that will prolong survival and improve quality of life.

The main objective of our study was to evaluate the role of magnetic resonance imaging (MRI), magnetic resonance spectroscopy (MRS) and transrectal ultrasound (TRUS) in detecting prostatic pathologies and staging of prostate cancer by correlating these methods with histopathological results.

Material/Methods:

The study is a cross-sectional diagnostic study performed in 66 patients with a high degree of clinical suspicion of prostatic pathology. All patients underwent TRUS, T1W, T2W, DWI, and 3D PRESSMRS sequences, and we also calculated ADC values and $\mathrm{Cho} \mathrm{Cr} / \mathrm{Cit}$ MRS ratios for all patients.

Results: $\quad$ Combination of MRI and MRS showed the highest diagnostic accuracy among the imaging modalities in detecting of prostatic neoplasm, followed by MRI, and then by TRUS. MRS plays a complementary role to MRI, by increasing its diagnostic accuracy. Due to a high cost, limited availability and increased scanning time, combination of MRI and MRS is currently not recommended as a first line investigation for detecting prostate neoplasms, hence USG (TRUS) remains the first line investigation due to its low cost, easy availability, time effectiveness and comparable efficacy.

Conclusions: $\quad$ MRI MRS has more diagnostic accuracy than MRI alone for detection of prostate pathologies. MRS, plays significant complementary role and should be included in the routine MR imaging protocols. MRI helps in diagnosis, localization, better tissue characterization and staging of prostate cancer. TRUS is easily available, cost effective and has comparable efficacy.

MeSH Keywords: $\quad$ Diffusion Magnetic Resonance Imaging • Magnetic Resonance Imaging • Magnetic Resonance Spectroscopy • Prostate • Prostatic Neoplasms • Ultrasound, High-Intensity Focused, Transrectal

PDF file: $\quad$ http://www.polradiol.com/abstract/index/idArt/903958 


\section{Background}

Prostate cancer $(\mathrm{PC})$ is the second most common cancer and the sixth leading cause of cancer related death among men worldwide. Moreover incidence of prostate cancer has been increasing over the years [1].

Prostate cancer is a potentially curable disease and combined effects of early detection and therapeutic intervention are likely causes of the observed reduction in prostate cancer mortality. The main goal of prostate cancer imaging is early diagnosis and more accurate disease characterization owing to the combined effects of anatomic, functional and molecular assessments [2,3]. PSA, digital rectal examination(DRE), TRUS-guided biopsy and computed tomography (CT) cannot correctly localize, stage or determine the volume, and aggressiveness of prostate cancer however MRI can be used for al those reasons [4,5].

\section{Material and Methods}

We performed a cross-sectional study to evaluate the efficacy of TRUS, MRI, MRI+MRS in various prostatic pathologies, with the main focus on detecting prostate cancer. A total of 66 patients were informed about the nature, and objective of the study and written informed consent was taken following an approval of an institutional ethics. All patients with a strong clinical suspicion of prostate pathologies (lower urinary tract symptoms like increased frequency of micturition, hesitancy, urgency and hard/enlarged prostate on digital rectal examination), enlarged prostate on ultrasound of the abdomen or raised PSA levels (>4 ng/ml) were included in the study.

Patients unwilling or unable (claustrophobic) to undergo MRI/MRS, with metallic hip implants or any other metallic implants or devices that might distort local magnetic fields and compromise the quality of MRI/MRS together with patients who underwent a recent prostatic biopsy were excluded from the study.

All patients underwent TRUS and later MRI with TIWI, T2WI, DWI and 3D PRESSMRS sequences; ADC values and Cho $\mathrm{Cr} /$ Cit ratios were calculated.

Machines Used - Ultrasonography (USG) machine - Mylab 50 and my lab 40, Corevision.

MRI - 1.5 Tesla (GE health care).

\section{Statistical methods}

The demographic data were obtained and summarized as absolute numbers and percentages. Patients were grouped according to the zonal distribution of lesions on USG and MRI, characterization of lesions on USG and MRI and histopathological interpretation of lesions. Histopathological results were compared with USG and MRI. Descriptive statistics like mean and standard deviation were obtained. The means across different assessment modalities were compared using one-way analysis of variance (ANOVA). The mean apparent diffusion coefficient $(A D C)$ value was obtained. The mean ADC across grades of lesions based on
Gleason score as well as prostatic lesions were compared using one-way ANOVA. The diagnostic accuracy of USG and MRI was obtained in comparison with histopathological findings. Receiver operating characteristics (ROC) analysis was performed for PSA and mean $\mathrm{Cho} \mathrm{Cr} / \mathrm{Cit}$ value with reference to histopathological findings to determine respective cut-off values. The diagnostic accuracy of such obtained cut-off values was also determined. Furthermore, the diagnostic accuracy of USG and MRI in predicting malignancy was determined. Also, the agreement of MRI with final of malignancy was assessed.

All the analyses were performed using SPSS version 20.0 (IBM Inc.)

\section{Results}

The largest proportion of patients in our study belonged to the age group 60-69 years constituting about $45.4 \%$ of all subjects.

The mean age in the benign category was 60.68 years, SD of 8.69 years, while the mean age in the malignant category was 68.97 years, SD of 7.97 years.

On MRI, the majority of lesions $45(68.2 \%)$ were hypointense on T2W sequences, while $21(31.8 \%)$ had a heterogeneous signal intensity on $\mathrm{T} 2 \mathrm{~W}$ images.

When MRS was combined with MRI, 24 patients (36.36\%) had a benign pathology and 42 patients $(63.64 \%)$ had a malignant pathology. Out of 42 malignant lesions, 21 lesions were peripheral zone neoplasms while 14 lesions were transitional zone neoplasms on MRI+MRS (Tables 1-4). The mean Cho $\mathrm{Cr}$ /Cit MRS values of benign, inflammatory and neoplastic lesions were significantly different (Table 1).

On histopathology, the majority of lesions $(62.12 \%)$ were diagnosed as malignant lesions. 6 patients $(9.09 \%)$ were diagnosed with chronic prostatitis, and 19 patients $(28.78 \%)$ were diagnosed with benign prostatic hyperplasia (Figure 1) Out of 41 patients with malignancies on histopathology, the majority had peripheral zone lesions.

The mean age of patients with malignant neoplastic disease $(68.97 \pm 7.97$ years) was significantly higher than that of patients with benign disease (60.68 \pm 8.69 years).

Out of 41 patients with a malignant pathology on histopathology, 32 were correctly identified as malignant on USG, 39 were correctly identified on MRI and 40 were correctly identified when MRS was added to MRI.

An inverse correlation was observed between Gleason scores and $\mathrm{ADC}$ values in pathologically proven neoplasms i.e. the lower the $\mathrm{ADC}$ value, the higher the tumor grade.

Thus, a combination of MRI+MRS (final diagnosis) showed the highest diagnostic accuracy among the imaging modalities for detection of prostatic neoplasms, followed by MRI and then by TRUS. MRS plays a complementary role to MRI, by increasing its diagnostic accuracy detecting prostate neoplasms (Tables 4, 5). 
Table 1. Mean Cho $\mathrm{Cr} /$ Cit values as per histopathological diagnosis.

\begin{tabular}{cccc}
\hline & & MRS [Mean \pm SD $(\mathbf{n})]$ & \\
\cline { 2 - 4 } Histopathology & Benign prostatic hyperplasia & Carcinoma & Prostatitis \\
\hline Mean Cho Cr/Cit value & $0.50 \pm 0.25(19)$ & $1.89 \pm 1.21(41)$ & $0.93 \pm 0.57(6)$ \\
\hline
\end{tabular}

P-value $<0.0001$ using one-way ANOVA.

Table 2. Comparison of final diagnosis (MRI+MRS) with histopathology.

\begin{tabular}{lccc}
\hline \multirow{2}{*}{ Final diagnosis } & \multicolumn{2}{c}{ Histopathology findings } & P-value* \\
\cline { 2 - 3 } & 23 & Carcinoma $(\mathbf{n}=\mathbf{4 1})$ & $<0.0001$ \\
\hline Non-malignant & 2 & 4 & \\
\hline Malignant & $97.56 \%$ & \\
\hline Sensitivity & $92.00 \%$ & \\
\hline Specificity & $95.24 \%$ & \\
\hline Positive predictive value & $95.83 \%$ & \\
\hline Negative predictive value & & \\
\hline
\end{tabular}

* Obtained using the chi-square test.

Table 3. Comparison of diffusion weighted imaging of MRI and histopathology finding in predicting malignancy.

\begin{tabular}{lccc}
\hline \multirow{2}{*}{ Histopathology } & \multicolumn{2}{c}{ DW } & P-value* \\
\cline { 2 - 3 } & Non-carcinoma & Carcinoma & $<0.0001$ (HS) \\
\hline Benign & 17 & 39 & \\
\hline Malignant & 2 & & \\
\hline Sensitivity & $82.98 \%$ & & \\
\hline Specificity & $89.47 \%$ & & \\
\hline Positive predictive value & $95.12 \%$ & & \\
\hline Negative predictive value & $68.00 \%$ & & \\
\hline
\end{tabular}

* Obtained using Chi-Square test; HS - highly significant.

Table 4. Comparison of diagnostic accuracy of different imaging modalities.

\begin{tabular}{lcccccc}
\hline \multicolumn{1}{c}{ Diagnostic parameter } & \multicolumn{2}{c}{ USG } & \multicolumn{2}{c}{ MRI } & \multicolumn{2}{c}{ Final diagnosis (MRI+MRS) } \\
\hline Sensitivity & $32 / 41$ & $78.05 \%$ & $39 / 41$ & $95.12 \%$ & $40 / 41$ & $97.56 \%$ \\
\hline Specificity & $22 / 25$ & $88.00 \%$ & $21 / 25$ & $84.00 \%$ & $23 / 25$ & $92.00 \%$ \\
\hline Positive predictive value & $32 / 35$ & $91.43 \%$ & $39 / 43$ & $90.70 \%$ & $40 / 42$ & $95.24 \%$ \\
\hline Negative predictive value & $22 / 31$ & $70.97 \%$ & $21 / 23$ & $91.30 \%$ & $23 / 24$ & $95.83 \%$ \\
\hline
\end{tabular}

On DCE MRI, areas demonstrating early, rapid and intense contrast uptake with subsequent plateau or wash-out phase were considered suspicious for the presence of malignancy. Some patients underwent DCE-MRI.

In our study, malignant cases showed an early wash-out and they were all malignant. Out of 4 cases that did not show a rapid wash-out, none turned out as malignant.

\section{Discussion}

\section{Prostate cancer screening}

Men who aged 50-60 years or older who present with lower urinary tract symptoms are now offered serum prostate-specific antigen (PSA) testing. The diagnostic 


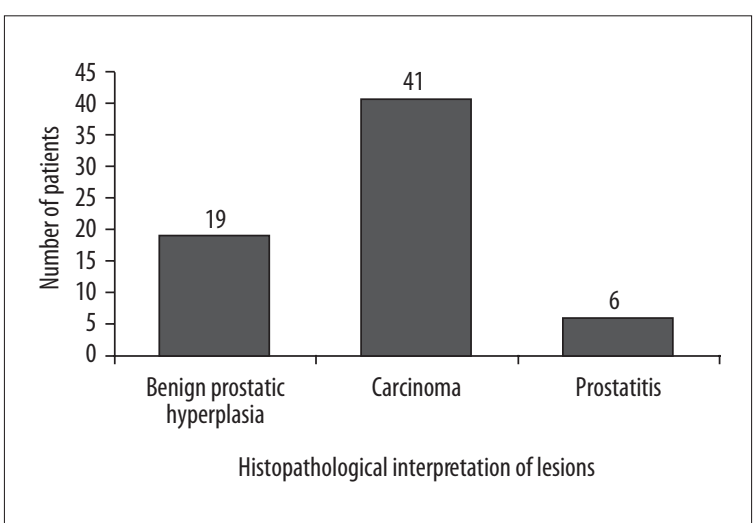

Figure 1. Bar diagram showing number of patients as per histopathological diagnosis of lesions.

evaluation is further offered by transabdominal or transrectal ultrasound.

\section{Serum PSA for prostate cancer screening and its impact}

PSA-based screening significantly increases the frequency of prostate cancer diagnosis at an early stage, which is important for further treatment. It is predicted that PSAbased screening can help to reduce the mortality by $20 \%$, but at a high cost of over diagnosis and overtreatment.

\section{Transrectal USG (TRUS) (Figure 2)}

It is a widely available, low-cost diagnostic tool used for morphological assessment of the prostate gland and its pathologies. However, it can neither reliably diagnose an intra-prostatic cancer nor detect its extra capsular extension in contrast to MRI (Figures 3,4). Although most cancers in the peripheral zone (PZ) are hypoechoic, some are hyperechoic. Others, including central gland cancers, are often difficult to diagnose. Color and power doppler imaging can further add information about the vascularity (and neovascularization) of the prostate and its lesions, however it is not $100 \%$ reliable. Therefore ultrasound can be used only for initial screening of prostate cancer. In case with equivocal findings, further tissue characterization is done by MRI. Currently ultrasound is used for initial screening and to guide prostatic biopsies. Most of the lesion can be diagnosed using transabdominal or transrectal ultrasound. If needed, further MRI with spectroscopy and contrast administration is done to obtain more accurate information. Some lesions may have been missed in our study due to a relatively low sensitivity of TRUS in detecting malignancy. Similar findings were also found by Sheth $S$ et al. who concluded that clinical stage A carcinomas may be difficult to detect on US, and findings are often nonspecific. Any suspicious peripheral zone lesions should undergo MRI or TRUS-guided biopsy before being diagnosed as malignant or benign [6].

In our study, on USG, the majority of patients (51.5\%) were diagnosed with malignant pathologies (34), while the rest had benign pathologies i.e. benign prostatic hyperplasia (26, $39.4 \%$ ) and prostatitis $(6,9.09 \%)$. Out of 25 patients with benign pathology on follow up, 22 were correctly diagnosed as a benign on USG follow-up. The sensitivity of USG was $78.05 \%$, specificity was $88.00 \%$, PPV was $91.43 \%$ and NPV was $70.97 \%$.

\section{TRUS-Guided Systematic Biopsy}

It has advantages, as it is real-time and easy of use. Often it is combined with MRI (TRUS-MRI fusion) for better localization, guidance, approach and morphological assessment of the lesion.

\section{Staging of prostate cancer - Gleason's score}

Definitive diagnosis is established only by transrectal ultrasound-guided biopsy (TRUS biopsy) and histopathological analysis for determination of cancer grade (Gleason score) and volume. The prognosis and choice of therapy is dependent on this information.

A significant relationship exists between tumor aggressiveness as denoted by Gleason score and the $\mathrm{Cho}+\mathrm{Cr} / \mathrm{Cit}$ ratio. In our study, an inverse correlation was observed between Gleason scores and ADC values in pathologically proven cases of neoplasms i.e. the lower the $\mathrm{ADC}$ value, the higher is the tumor grade.

\section{Magnetic resonance imaging (Figures 3, 5-7)}

Magnetic resonance imaging (MRI) is an imaging modality which significant information on the anatomy, pathology and extent of prostate pathologies. MRI has many advantages that make it a favorable modality (e.g., high contrast resolution, ability to obtain images in any plane i.e. multiplanar imaging, no ionizing radiation, and safety of using particulate contrast media rather than those containing iodine).

Table 5. Statistical significance testing of various diagnostic parameters between imaging modalities.

\begin{tabular}{lccc}
\hline \multirow{2}{*}{ Diagnostic parameter } & \multicolumn{4}{c}{ P-value } \\
\cline { 2 - 4 } & USG vs. MRI & USG vs. final diagnosis (MRI+MRS) & MRI vs. final diagnosis (MRI+MRS) \\
\hline Sensitivity & 0.052 & $0.018(S)$ & 0.999 \\
\hline Specificity & 0.999 & 0.999 & 0.663 \\
\hline Positive predictive value & 0.999 & 0.833 & 0.694 \\
\hline Negative predictive value & 0.135 & $0.043(\mathrm{~S})$ & 0.969 \\
\hline * Obtained using the z-test for proportions. & & & \\
\hline \hline
\end{tabular}




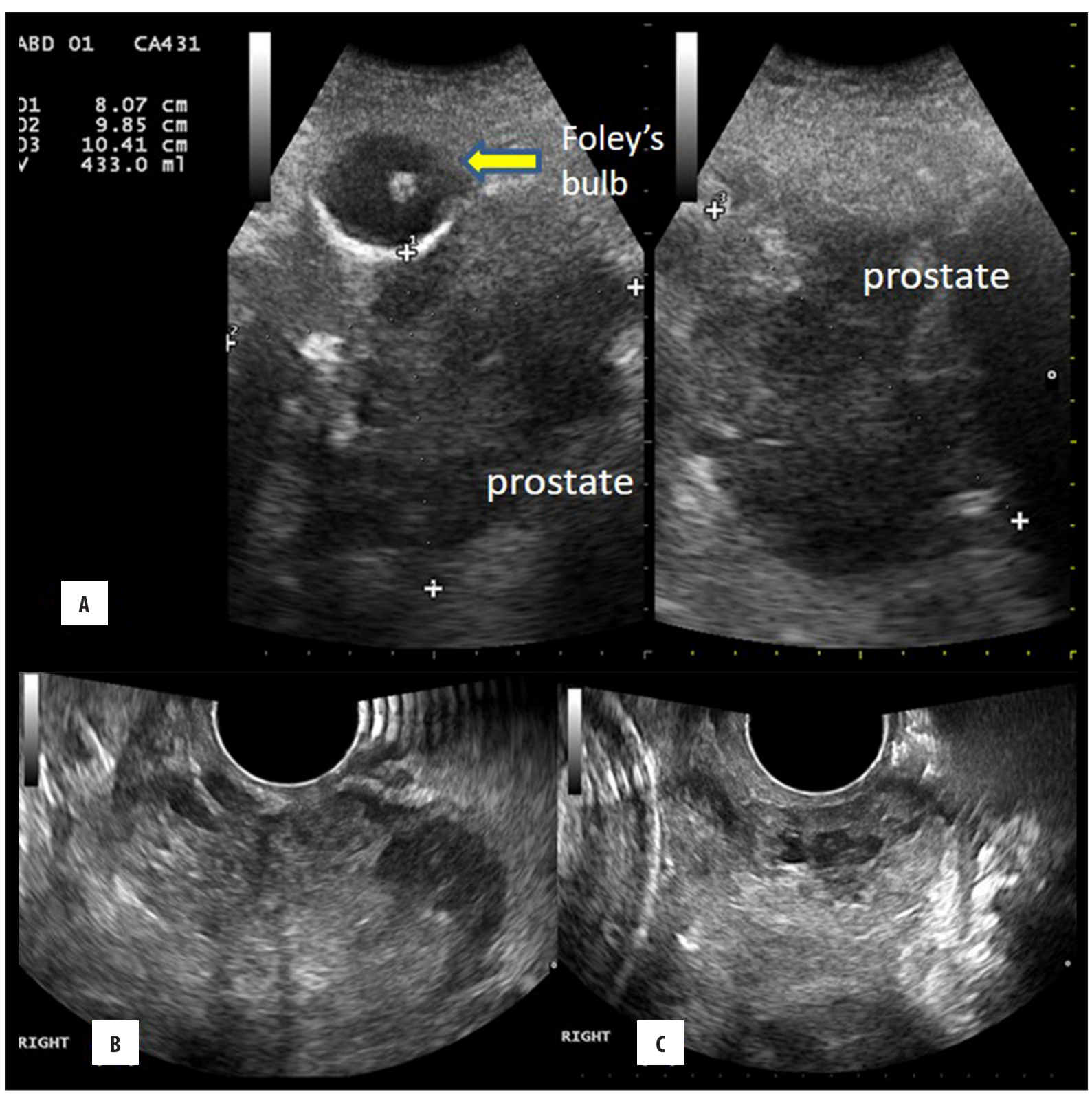

Figure 2. Ultrasonography and TRUS in a 85 year male with giant prostatomegaly (A) transabdominal USG showing prostate of volume 433 cC (B) TRUS showing enlarged right lobe of prostate (C) TRUS showing enlarged prostate.

In our study, lesions were identified and diagnosed using TRUS, TIW, T2W, DWI and 3D PRESSMRS sequences. Then, the lesions were characterized based on their appearance and the $\mathrm{Cho}+\mathrm{Cr} /$ Cit ratio of these lesions was calculated from the corresponding metabolite peaks. Lesions were localized to a particular zone.

\section{Diffusion-weighted imaging}

DWI exploits the property of constant Brownian motion of water molecules in tissues. This property is affected by increased cellularity, tissue organization, extracellular space, and integrity of cell membranes. Prostate cancer foci are composed of tightly packed cellular elements with reduced extracellular space, which can be visualized on DWI images as areas of restricted diffusion (high signal intensity), with corresponding low signal intensity on apparent diffusion coefficient (ADC) maps. Diffusionweighted imaging demonstrates restriction of diffusion and reduction of apparent diffusion coefficient values in neoplastic tissue. This technique requires short acquisition time and provides high contrast resolution between neoplastic and normal tissues.

\section{Magnetic resonance spectroscopy}

Magnetic resonance spectroscopy, is an adjuvant to magnetic resonance imaging, which offers a level of tissue characterization that can match histological and biochemical diagnosis. MR spectroscopy is also known as chemical shift imaging.

One such application is in the field of prostate neoplasm. MRS when combined with MRI has a potential to detect, 


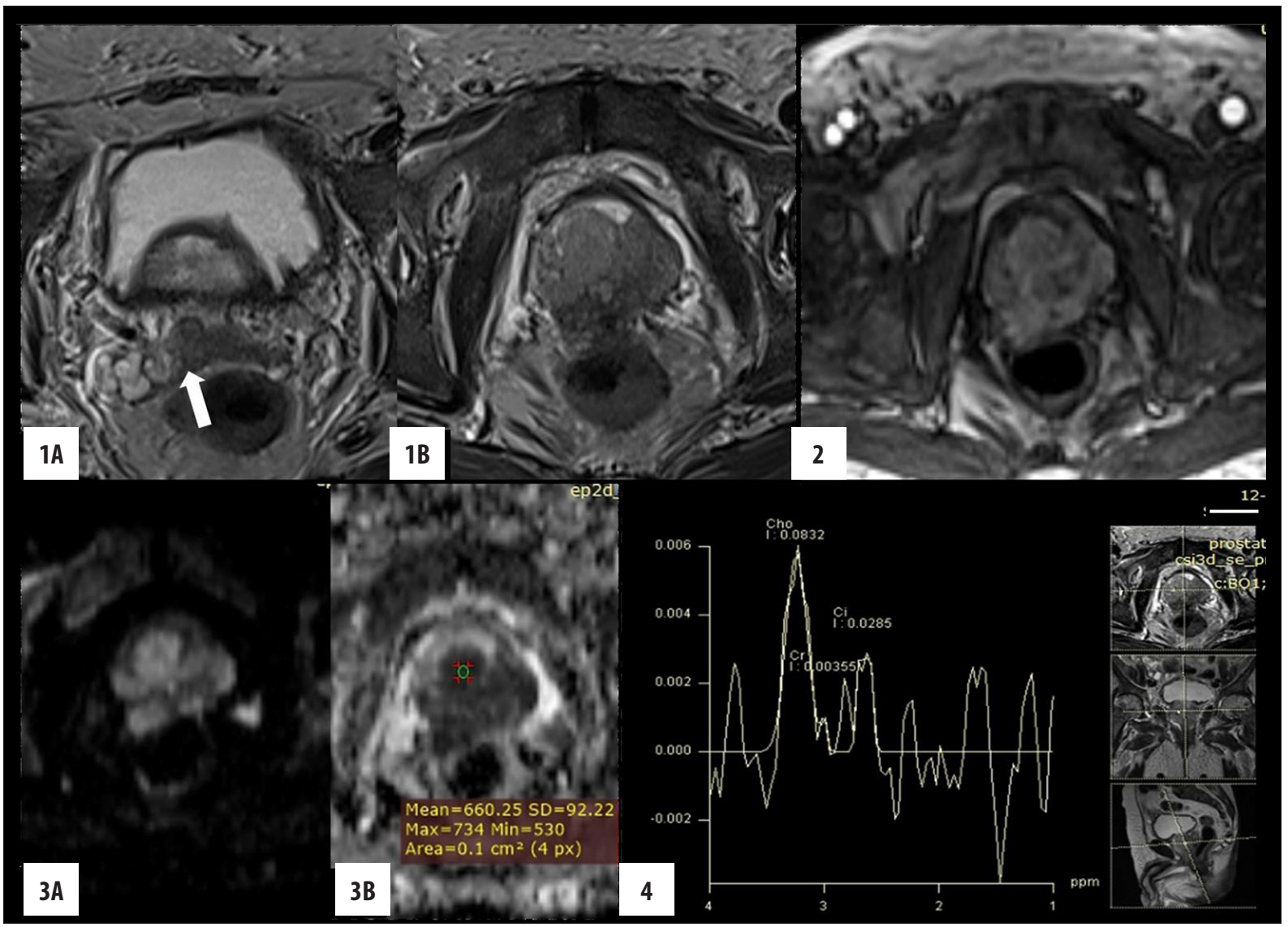

Figure 3. Ca prostate with extracapsular extension and involvement of seminal vesicle. 1. T2W axial image showing (1A) hypointense signal in the root of right seminal vesicle(arrow) (1B) diffuse hypointense signal in both TZ and PZ with ECE. 2. DCE- MRI showing early arterial enhancement. 3. (A) Area of restricted diffusion on DWI with (B) corresponding hypointensity on ADC map. 4. MRS showing raised choline and decrease citrate peak.

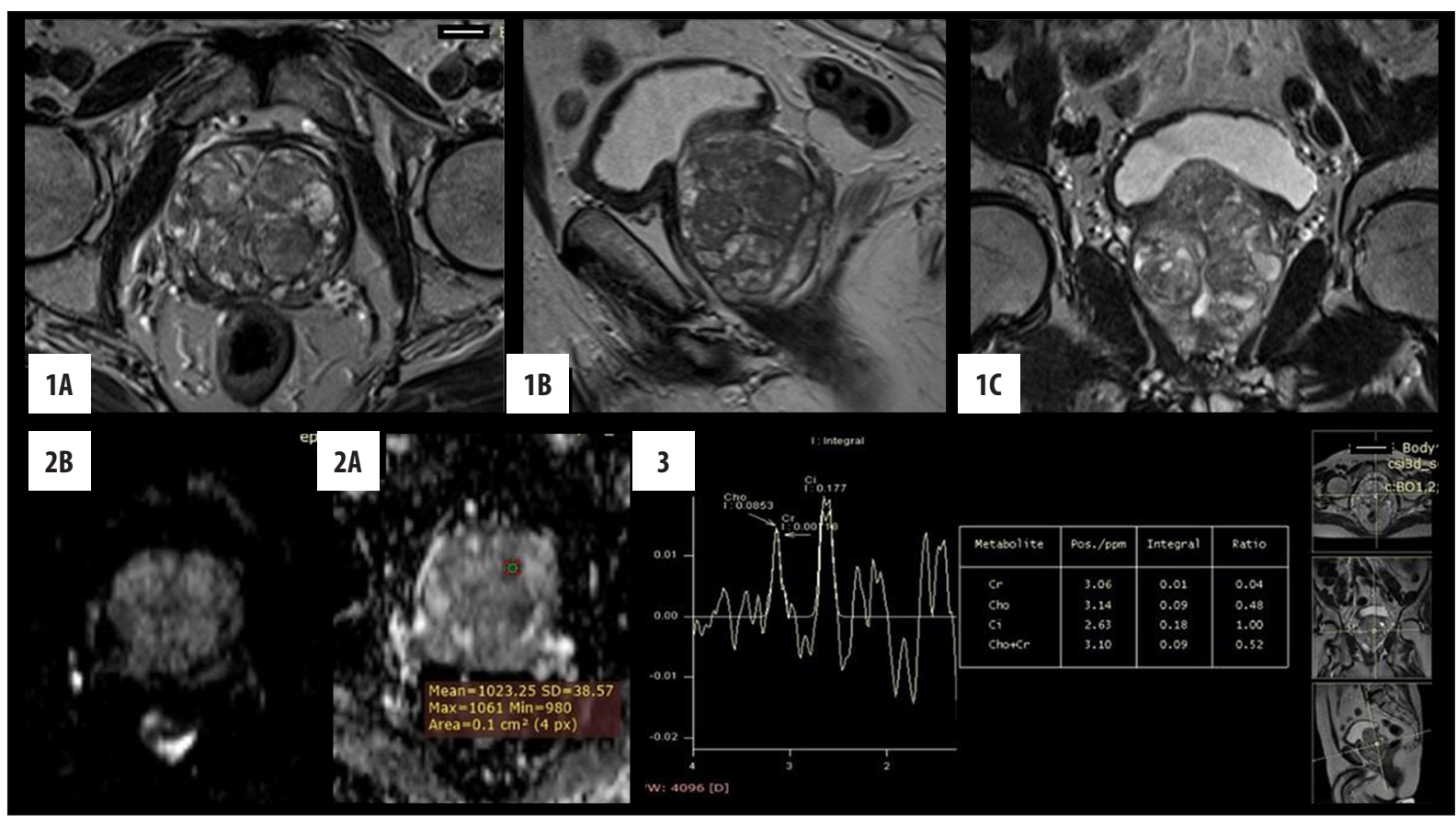

Figure 4. BPH.1. T2W images (1A) axial, (1B) sagittal and (1C) coronal showing heterogeneous signal in central gland with multiple well circumscribed nodules. 2. (2A)DWI and (2B) ADC map do not show restricted diffusion mean $A D C$ value is $1023 \times 10^{-6} \mathrm{~mm}^{2} / \mathrm{s}$. 3. MR spectroscopy: $\mathrm{Cho}+\mathrm{Cr} / \mathrm{Ci}$ ratio is normal 


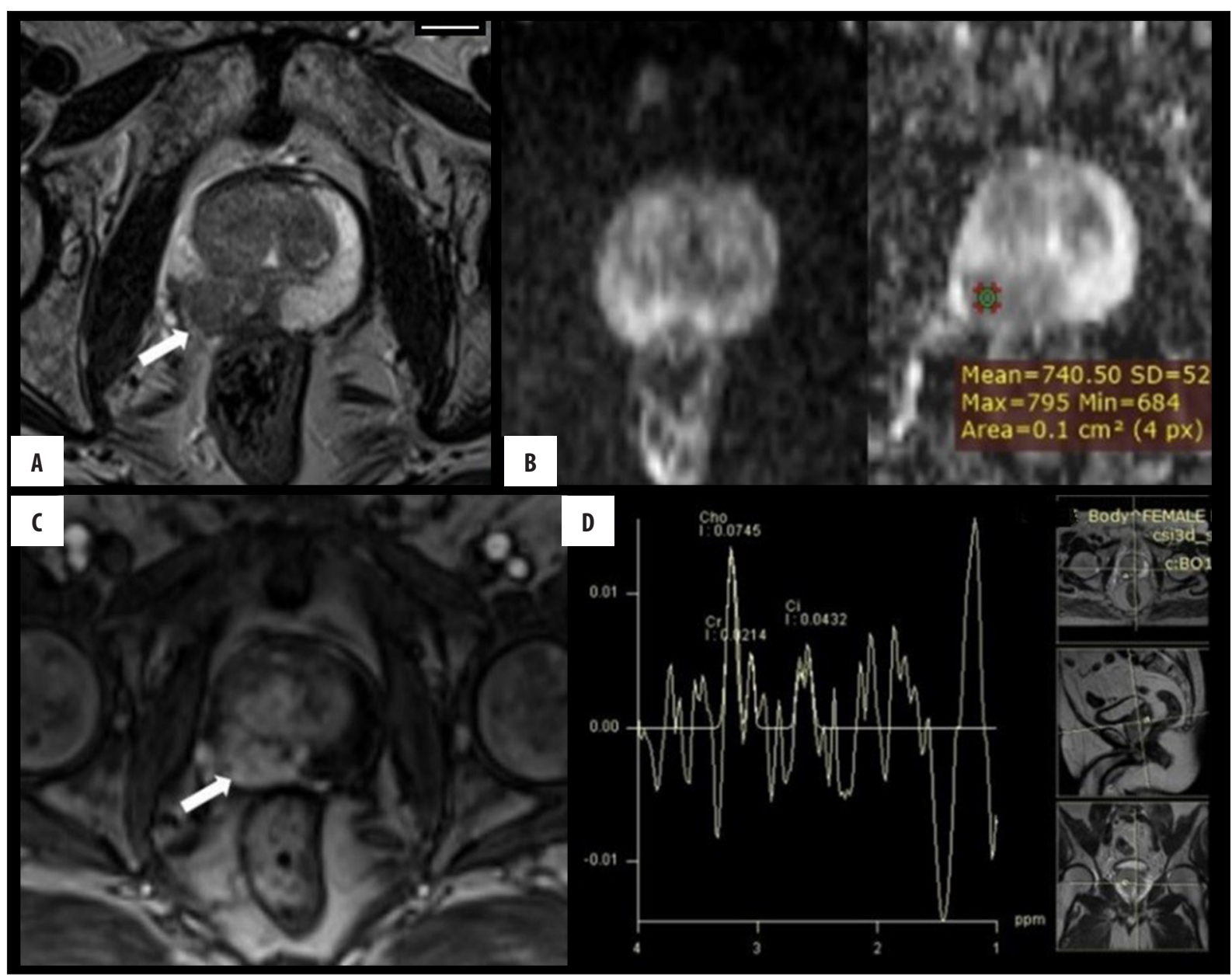

Figure 5. Peripheral Zone Carcinoma. (A) T2W axial image showing focal circumscribed area of moderate hypointensity (arrow) in right peripheral zone. (B) Corresponding area showing restricted diffusion on DWI and hypointensity on ADC map. (C) Early arterial enhancement on DCE noted in the corresponding area(arrow). (D) MRS shows elevated choline peak and reduced citrate peak with voxel placement in the suspicious area.

characterize and localize lesions, and it can differentiate between benign and malignant lesions and determine the stage and aggressiveness of prostate neoplasms. In addition, it can also predict the prognosis and help in management of prostate lesions. It thus plays a complementary role to MRI in patients with prostate neoplasms. MR spectroscopy, which depicts a higher ratio of choline and creatine to citrate in neoplastic tissue than in normal tissue, is generally accepted. The technique also allows detection of prostate neoplasms in the transitional zone. However, it requires a long acquisition time, does not directly depict the periprostatic area, and is frequently affected by artifacts.

Lesions were detected and localized on MRS by assessing the maximum $\mathrm{Cho}+\mathrm{Cr} / \mathrm{Cit}$ ratio. The maximum $\mathrm{Cho}+\mathrm{Cr} / \mathrm{Cit}$ ratio was also used by Kobus et al. [7].

MRI and MRS localized lesions to respective zones with equal efficacy. However, MRS played a complementary role to MRI in localization of lesions within zones. A study by Scheidler et al. [8] showed that the combined use of MR imaging and MR spectroscopy improves the detection of tumors within the peripheral zone.
In our study, patients diagnosed with benign prostatic hyperplasia on histopathology had a mean $\mathrm{Cho}+\mathrm{Cr} / \mathrm{Cit}$ value of $0.50 \pm 0.25$ (19 patients). Patients with a malignant pathology had a mean Cho+Cr/Cit value of $1.89 \pm 1.21$ (4l patients) and in patients with prostatitis it was $093 \pm 0.57$ (6 patients). These findings were in line with previous studies by Kurhanewicz et al. [9] (mean Cho+Cr/Cit ratio in malignancy of 2.1 \pm 1.3 and in BPH of $0.61 \pm 0.21$ ) and Kim JK et al. [10] (mean Cho $+\mathrm{Cr} / \mathrm{Cit}$ in malignancy of $1.89 \pm 1.21$ (41 patients) and in $\mathrm{BPH}$ of $0.50 \pm 0.25$ (19 patients). The mean value of $\mathrm{Cho}+\mathrm{Cr} / \mathrm{Cit}$ for malignant lesions in our study was significantly higher than that for benign lesions, which is in line with studies by Shukla-Dave A et al. [11], Kurhanewicz et al. [9], Kim JK et al. [10] and Kurhanewicz et al. [12].

Kobus et al. [7] found a significant correlation between aggressiveness and maximum $\mathrm{Cho}+\mathrm{Cr} / \mathrm{Cit}$ ratios and they stated that MRS offered a potential for a noninvasive in vivo assessment of prostate neoplasm aggressiveness. Similarly, Zakian et al. [13] observed that $\mathrm{Cho}+\mathrm{Cr} / \mathrm{Cit}$ ratios of prostate tumors and tumor volume correlated with Gleason scores, and that there was a trend toward increasing $\mathrm{Cho}+\mathrm{Cr} /$ Cit ratios with increasing Gleason scores in lesions identified correctly with MRS 


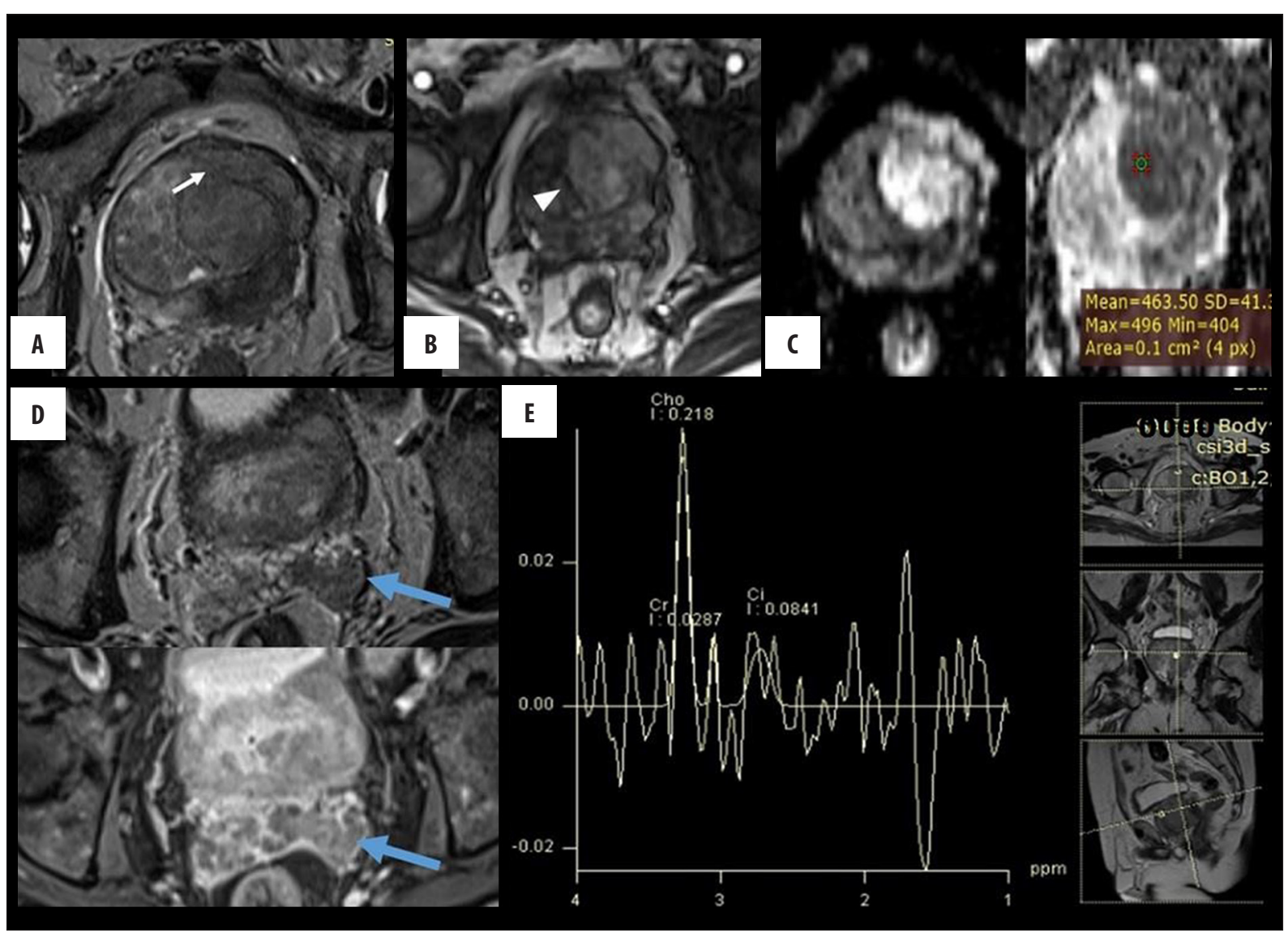

Figure 6. Transition zone Carcinoma. (A) T2W axial image showing ill-defined hypointensity involving predominantly left transition zone with extracapsular extension (arrow). (B) DCE shows early arterial enhancement in the corresponding area. (C) Focal area of restricted diffusion and $A D C$ hypointensity in the corresponding area. (D) Loss of normal T2W hyperintense signal in left seminal vesicle (above) with post contrast enhancement(below). (E) MRS shows elevated choline and reduced citrate peak.

In our study, out of 41 patients with a malignant pathology on histopathology, 40 were correctly diagnosed by MRI+MRS in comparison to 39 on MRI and 32 on USG. However, similarly to other modalities, the combination of MRI+MRS identified 23 out of 25 benign cases. Thus, the combination of MRI+MRS correctly identified 2 additional cases of malignancy in comparison to MRI alone and 8 additional cases of malignancy in comparison to USG alone, reflecting that it had the highest sensitivity (97.56\%) and specificity (92\%) among all the imaging modalities in our study. Out of 2 cases that were wrongly diagnosed as malignant on MRI+MRS, 2 cases were diagnosed as BPH on histopathology. PPV and NPV was 95.24\% and 95.83\%, respectively, when MRS was added to MRI. Scheidler et al. [8] found sensitivity and specificity for neoplasm detection of $91 \%$ and $95 \%$, respectively for a combined use of MR spectroscopy and MR imaging, in comparison to $77 \%$ sensitivity of MRI alone. Thus, they observed that addition of 3D MRS to MRI provided a better detection of prostate neoplasms with sensitivity and specificity higher than in the case of MRI alone. Squillaci et al. [14] observed that sensitivity of neoplasm detection was increased on MRS (89\%) in comparison to MRI (85\%), while specificity of either modality remained the same

\section{Dynamic contrast-enhanced MRI}

The basic principle of DCE-MRI is related to tumor angiogenesis. Any tumor $>2 \mathrm{~mm}$ inevitably shows angiogenesis. Prostate cancer, due to the expression of vascular endothelial growth factor (VEGF), is no exception. There is a difference between the interstitial space of cancerous tissue and normal tissue. Due to large interstitial spaces in the cancerous tissue, there is a difference in the concentration of intravenous contrast material between intravascular and extravascular spaces, which accentuates contrast transfer through vascular walls and thus results in unique enhancement patterns of strong early enhancement and rapid washout of contrast. DCE-MRI has a high accuracy and sensitivity. On DCE MRI, areas demonstrating early, rapid, and intense contrast uptake with subsequent plateau or wash-out phase were considered suspicious for the presence of malignancy.

\section{Multi-parametric or combined approach of different MRI sequences to reach final diagnosis}

In our study, we aimed to determine the role of $1.5 \mathrm{~T}$ magnetic resonance imaging and transrectal ultrasound in the evaluation of prostatic pathologies and in the detection and staging of prostate neoplasm. We have correlated our 


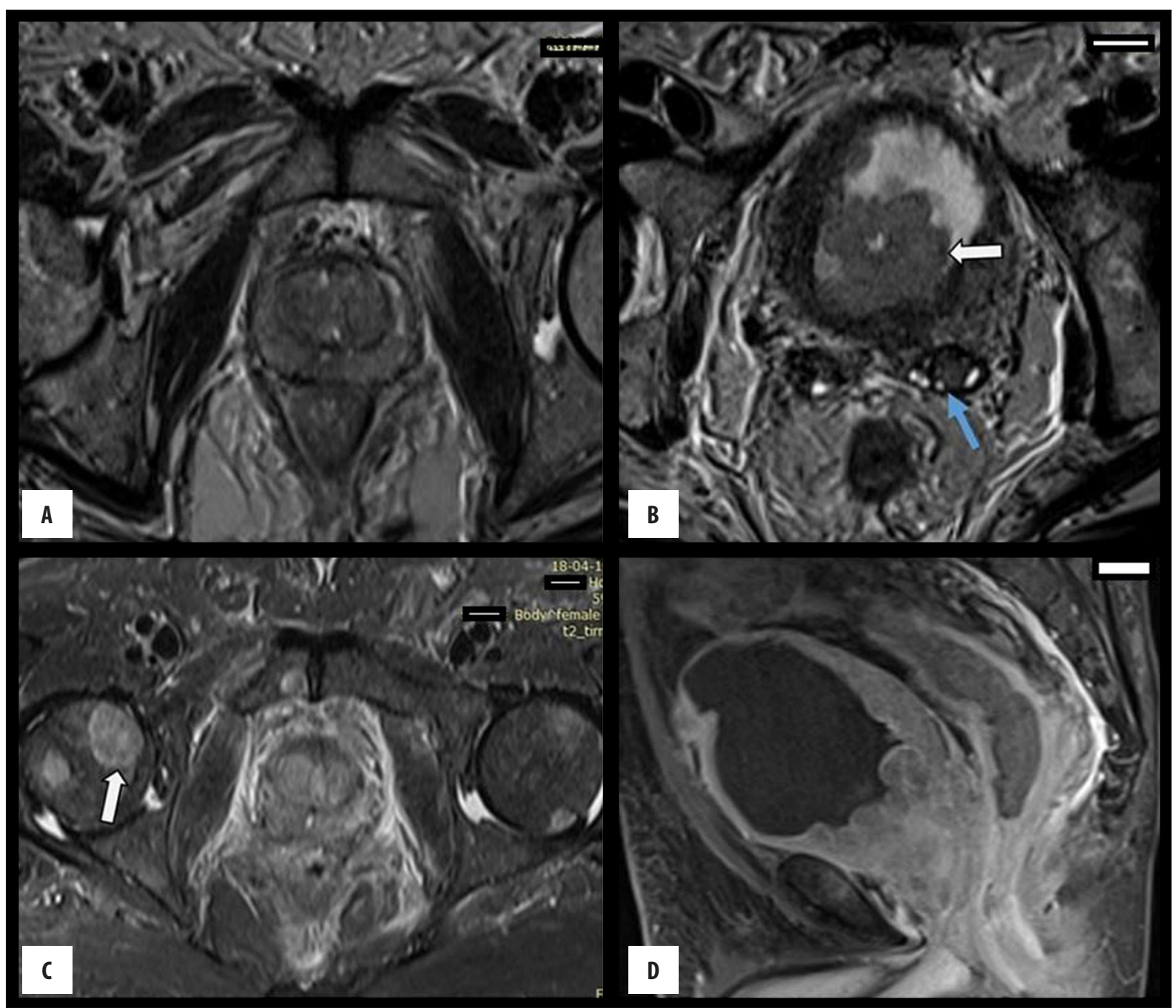

Figure 7. Ca Prostate with seminal vesicle involvement, bladder base invasion and bone metastases. (A) T2W axial images shows diffuse hypointense signal in both PZ and TZ. (B) T2W Axial image showing hypointense signal in left seminal vesicle( blue arrow) and polypoidal growth in the bladder base. (C) STIR axial image showing multiple focal areas of hyperintense signal in pelvic bones( head of femur arrow and pubic symphysis). (D) T1FS post contrast sagittal image showing heterogeneous enhancement of prostate with involvement of the adjacent bladder base .

findings with biopsy and post-operative histopathological diagnosis.

Abscesses also show restriction of diffusion just like most prostate cancers. However, they appear hyperintense on T2WI and show only peripheral enhancement instead of heterogonous or diffuse enhancement of neoplastic tissue [15].

In our study, there was 1 case diagnosed as non-malignant on plain MRI without spectroscopy and as malignant on MRI with spectroscopy. Two cases were diagnosed as malignant on MRI without spectroscopy but as non-malignant on MRI with spectroscopy, as compared to histopathology.

Thus, a combination approach consisting of T2WI, DWI, $\mathrm{ADC}$ values, DCE-MRI, and spectroscopy can better diagnose, detect, and localize prostatic lesions.

\section{Conclusions}

The present study was undertaken to determine the role of transrectal ultrasound (TRUS), magnetic resonance imaging (MRI), and magnetic resonance spectroscopy (MRS) in the evaluation of prostatic pathologies, mainly prostate neoplasms. TRUS, MRI, and MRS scans were reviewed, the prostatic lesions were identified and characterized, and the results were correlated with histopathological findings.

Due to a high cost, limited availability, and prolonged scanning time, MRI and MRS is currently not recommended as a first-line investigation for detecting prostate neoplasms. USG (trans-abdominal and transrectal ultrasonography) remains the first-line investigation due to its low cost, easy reproducibility, widespread availability, time effectiveness, and comparable efficacy. However, in patients with equivocal findings on USG and a high clinical suspicion of neoplasm, MRI with spectroscopy is advised for more accurate tumor diagnosis and staging. 
MRI with MRS is highly effective in the detection, diagnosis, localization, characterization, and staging of clinically significant prostate cancer and other prostatic lesions. It also has potential applications for tumor staging and can predict the aggressiveness of tumors. The combination of MRI+MRS has an improved diagnostic accuracy in comparison to MRI alone for the detection of prostate neoplasms. MRS, though time consuming, plays a very important adjuvant role to MRI in the evaluation of prostatic pathologies and prostatic tumors, and it should be included in routine MR imaging protocols to evaluate prostatic pathologies.

\section{Conflicts of interest}

There are no conflicts of interest.

\section{Acknowledgement}

I acknowledge the help of Karanbir bajwa, Divya Kant, Purva Agrawal, Prashant Mudliar,Vikrant Bhende.

\section{References:}

1. Jain S, Saxena S, Kumar A: Epidemiology of prostate cancer in India. Meta Gene, 2014; 2: 596-605

2. Polascik TJ, Oesterling JE, Partin AW: Prostate specific antigen: A decade of discovery-what we have learned and where we are going. J Urol, 1999; 162(2): 293-306

3. Hricak H, Choyke PL, Eberhardt SC et al: Imaging prostate cancer: A multidisciplinary perspective. Radiology, 2007; 243(1): 28-53. Review. Erratum in: Radiology, 2007; 245(1): 302

4. Steyn JH, Smith FW: Nuclear magnetic resonance imaging of the prostate. Br J Urol, 1982; 54: 726-28

5. Coakley FV, Qayyum A, Kurhanewicz J: Magnetic resonance imaging and spectroscopic imaging of prostate cancer. J Urol, 2003; 170: S69-75

6. Sheth S, Hamper UM, Walsh PC et al: Stage A adenocarcinoma of the prostate: Transrectal US and sonographic-pathologic correlation. Radiology, 1991; 179(1): 35-39

7. Kobus T, Vos PC, Hambrock T et al: Prostate cancer aggressiveness: In vivo assessment of MR spectroscopy and diffusion-weighted imaging at 3 T. Radiology, 2012; 265(2): 457-67

8. Scheidler J, Hricak H, Vigneron DB et al: Prostate cancer: Localization with three-dimensional proton MR spectroscopic imaging - clinicopathologic study 1. Radiology, 1999; 213(2): 473-80
9. Kurhanewicz J, Vigneron DB, Hricak $\mathrm{H}$ et al: Three-dimensional H-1 MR spectroscopic imaging of the in situ human prostate with high

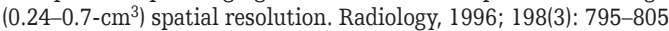

10. Kim JK, Kim DY, Lee YH et al: In vivo differential diagnosis of prostate cancer and benign prostatic hyperplasia: Localized proton magnetic resonance spectroscopy using external-body surface coil. Magn Reson Imaging, 1998; 16(10): 1281-88

11. Shukla-Dave A, Hricak H, Moskowitz C et al: Detection of prostate cancer with MR spectroscopic imaging: An expanded paradigm incorporating polyamines 1. Radiology, 2007; 245(2): 499-506

12. Kurhanewicz J, Vigneron DB, Nelson SJ et al: Citrate as an in vivo marker to discriminate prostate cancer from benign prostatic hyperplasia and normal prostate peripheral zone: Detection via localized proton spectroscopy. Urology, 1995; 45(3): 459-66

13. Zakian KL, Sircar K, Hricak H et al: Correlation of proton MR spectroscopic imaging with gleason score based on step-section pathologic analysis after radical prostatectomy 1. Radiology, 2005; 234(3): 804-14

14. Squillaci E, Manenti G, Mancino S et al: MR spectroscopy of prostate cancer. Initial clinical experience. J Exp Clin Cancer Res, 2005; 24(4): $523-30$

15. Singh P, Yadav MK, Singh SK et al: Case series: Diffusion weighted MRI appearance in prostatic abscess. Indian J Radiol Imaging, 2011; 21(1): $46-48$ 